OPEN ACCESS

Edited by:

Christine Marosi,

Medical University of Vienna, Austria

Reviewed by:

Alessia Pellerino,

University Hospital of the City of

Health and Science of Turin, Italy

Martha Nowosielski,

Innsbruck Medical University, Austria

${ }^{*}$ Correspondence:

Tracy L. Luks

Tracy.Luks@ucsf.edu

Specialty section:

This article was submitted to Neuro-Oncology and Neurosurgical

Oncology,

a section of the journal

Frontiers in Neurology

Received: 02 September 2021 Accepted: 20 December 2021

Published: 28 January 2022

Citation:

Luks TL, Villanueva-Meyer JE,

Weyer-Jamora C, Gehring K,

Jakary A, Hervey-Jumper SL, Braunstein SE, Bracci PM, Brie MS, Smith EM, Chang SM and Taylor JW

(2022) T2 FLAIR Hyperintensity

Volume Is Associated With Cognitive

Function and Quality of Life in Clinically

Stable Patients With Lower Grade

Gliomas. Front. Neurol. 12:769345.

doi: 10.3389/fneur.2021.769345

\section{T2 FLAIR Hyperintensity Volume Is Associated With Cognitive Function and Quality of Life in Clinically Stable Patients With Lower Grade Gliomas}

\author{
Tracy L. Luks ${ }^{1 *}$, Javier E. Villanueva-Meyer ${ }^{1}$, Christina Weyer-Jamora ${ }^{2,3}$, Karin Gehring ${ }^{4,5}$, \\ Angela Jakary ${ }^{1}$, Shawn L. Hervey-Jumper ${ }^{3}$, Steve E. Braunstein ${ }^{6}$, Paige M. Bracci ${ }^{7}$, \\ Melissa S. Brie ${ }^{2,3}$, Ellen M. Smith ${ }^{3}$, Susan M. Chang ${ }^{3}$ and Jennie W. Taylor ${ }^{3}$ \\ ${ }^{1}$ Department of Radiology and Biomedical Imaging, University of California, San Francisco, San Francisco, CA, \\ United States, ${ }^{2}$ Zuckerberg San Francisco General Hospital, San Francisco, CA, United States, ${ }^{3}$ Department of Neurological \\ Surgery, University of California, San Francisco, San Francisco, CA, United States, ${ }^{4}$ Department of Neurosurgery, \\ Elisabeth-TweeSteden Hospital, Tilburg, Netherlands, ${ }^{5}$ Department of Cognitive Neuropsychology, Tillburg University, Tilburg, \\ Netherlands, ${ }^{6}$ Department of Radiation Oncology, University of California, San Francisco, San Francisco, CA, United States, \\ ${ }^{7}$ Department of Epidemiology and Biostatistics, University of California, San Francisco, San Francisco, CA, United States
}

Survival outcomes for patients with lower grade gliomas (LrGG) continue to improve. However, damage caused both by tumor growth and by the consequences of treatment often leads to significantly impaired cognitive function and quality of life (QoL). While neuropsychological testing is not routine, serial clinical MRls are standard of care for patients with LrGG. Thus, having a greater understanding of MRI indicators of cognitive and QoL impairment risk could be beneficial to patients and clinicians. In this work we sought to test the hypothesis that in clinically stable LrGG patients, T2 FLAIR hyperintensity volumes at the time of cognitive assessment are associated with impairments of cognitive function and QoL and could be used to help identify patients for cognitive and QoL assessments and interventions. We performed anatomical MR imaging, cognitive testing and QoL assessments cross-sectionally in 30 clinically stable grade 2 and 3 glioma patients with subjective cognitive concerns who were 6 or more months post-treatment. Larger post-surgical T2 FLAIR volume at testing was significantly associated with lower cognitive performance, while pre-surgical tumor volume was not. Older patients had lower cognitive performance than younger patients, even after accounting for normal age-related declines in performance. Patients with Astrocytoma, IDH mutant LrGGs were more likely to show lower cognitive performance than patients with Oligodendroglioma, IDH mutant 1p19q co-deleted LrGGs. Previous treatment with combined radiation and chemotherapy was associated with poorer self-reported QoL, including self-reported cognitive function. This study demonstrates the importance of appreciating that LrGG patients may experience impairments in cognitive function and QoL over their disease course, including during periods of otherwise sustained clinical stability. Imaging factors can be helpful in identifying vulnerable patients who would benefit from cognitive assessment and rehabilitation.

Keywords: glioma, neuroimaging, neuro-oncology, neuropsychology, cognition 


\section{INTRODUCTION}

Survival outcomes for patients with lower grade (WHO grade 2 and 3) gliomas ( $\mathrm{LrGG}$ ) continue to improve as diagnosis and treatment evolve, with current median survival of 5-15 years (1). However, for patients with LrGG, tissue damage caused by tumor growth and by the consequences of treatment often leads to significantly impaired cognitive function (2-7). These cognitive impairments frequently have a negative impact on patients' QoL (8).

Within the LrGG patient population, there is high variability in the prevalence of cognitive and QoL impairment, and individual differences in the specific cognitive domains that are affected (9-15), although impairments in executive function, memory, and attention are most prevalent (11). Thus, it is challenging for clinicians to anticipate the extent to which a patient's cognition and QoL will be impacted. The timing of these impairments over the disease course is also unclear. Some reports suggest that cognitive impairments at the time of diagnosis resolve after acute recovery from surgery (e.g. within 3 months) $(16,17)$. However, others argue that practice effects are not always considered in those reports of improvement (18), and that while some recovery does occur, many patients remain impaired in specific cognitive functions and QoL domains (12). Additional impairment may emerge over the period of clinical stability following treatment due to longer term effects of radiation therapy, chemotherapy, and subtle tumor growth (19-23). Furthermore, impairments that were present since diagnosis or surgery may only become apparent to patients as they complete treatment and begin to resume the trajectories of their "normal" lives, re-encountering challenges in work, home, or family life.

The heterogeneity in the prevalence, nature and timing of impairments in cognitive function and QoL in patients with LrGG makes it difficult for clinicians, patients and caregivers to predict, monitor for, and respond to these impairments. The inclusion of cognitive function and QoL in LrGG patient treatment planning is of increasing interest as neuropsychological rehabilitation options expand (24). While neuropsychological testing is not routine, serial follow-up clinical MRIs are standard of care for patients with LrGG, so MRI indicators of cognitive and QoL impairment risk could be beneficial to patients and clinicians. For example, larger pre-surgical tumor volumes at diagnosis have been associated with poorer pre-surgical cognitive function and QoL in glioma $(7,25,26)$, but the association between these pre-surgical volumes and longer term cognitive outcomes is unclear (27). To our knowledge, the associations between imaging measurements, cognitive function, and QoL in stable LrGG patients have not been investigated together in one study. In this work we sought to test the hypothesis that in clinically stable LrGG patients, T2 FLAIR hyperintensity volumes at the time of cognitive assessment are associated with impairments of cognitive function and QoL, and could be used to help identify patients for cognitive and QoL assessments and interventions.

\section{METHODS}

\section{Patients}

Study participants were histologically confirmed LrGG (grade 2 and 3 glioma) patients who met the following inclusion criteria: (1) clinically stable and off treatment (i.e. surgery, radiation, chemotherapy) for at least 6 months, (2) expressed subjective cognitive concerns in discussion with their referring neuro-oncologist, (3) over 18 years old, (4) have a Karnofsky performance status (KPS) $\geq 70$, (5) be fluent in English, (6) off steroids, and (7) have cognitive and motor function sufficient to complete the cognitive and QoL assessments. The restriction of referral based on subjective cognitive concerns was implemented because, in our experience, patients with cognitive concerns are more interested in participating in research that requires lengthy cognitive testing. Therefore, this inclusion criteria aimed to optimize the time referring neurooncologists were requested to spend discussing this study with patients.

Clinical stability was determined by the referring physician, based on lack of significant current growth in T2 FLAIR hyperintensity or T1 contrast-enhancing lesion, or worsening focal neurological symptoms. There was no upward limit on the time between completed treatment and enrollment, or the number or types of previous treatments. All participants gave written informed consent, and ethical approval was granted by the UCSF Institutional Review Board, in compliance with the Helsinki Declaration.

\section{Cognitive and QoL Assessment}

Patients completed a one-hour computerized battery of standardized cognitive tests and QoL assessments within 1 month of a standard of care neuro-oncology appointment and MR imaging. The cognitive assessment included the adult Cognitive Domain tasks of the NIH Toolbox (http:// www.nihtoolbox.org/Pages/default.aspx), a package that is validated, age-normed (28), and used in previous assessments of cognitive function in glioma patients $(29,30)$. The following cognitive domains were tested: spatial and episodic memory, processing speed, attention and executive functions, inhibition and attention, language comprehension and vocabulary, working memory, and reading and speech (see Table 1 for test details).

The NIH Toolbox provides raw scores and age-corrected scores. Because glioma patients vary widely in age, we used the age-corrected scores to control for the effects of normal aging on cognitive function. In addition to the 7 individual test scores, the NIH Toolbox provides a crystallized cognition composite score (based on vocabulary and reading recognition); a fluid cognition composite score (based on processing speed, attention, and working memory); and a total cognition score.

Crystallized abilities depend on knowledge and skills acquired through culture and education, often in childhood and adolescence, and are assessed with tasks of language comprehension, speech, vocabulary, and reading (31). Fluid abilities involve dynamic, flexible problem-solving, and are 
TABLE 1 | Cognitive and quality of life assessment.

\begin{tabular}{|c|c|c|}
\hline Test name & Domain tested & Composite score \\
\hline $\begin{array}{l}\text { Pattern comparison } \\
\text { processing speed }\end{array}$ & Processing speed & Fluid cognition \\
\hline $\begin{array}{l}\text { Flanker inhibitory control } \\
\text { and attention }\end{array}$ & Inhibition and attention & \\
\hline $\begin{array}{l}\text { Dimensional change card } \\
\text { sort }\end{array}$ & $\begin{array}{l}\text { Attention and executive } \\
\text { functions }\end{array}$ & \\
\hline Picture sequence memory & $\begin{array}{l}\text { Spatial and episodic } \\
\text { memory }\end{array}$ & \\
\hline List sorting working memory & Working memory & \\
\hline Oral reading recognition & Reading and speech & $\begin{array}{l}\text { Crystallized } \\
\text { cognition }\end{array}$ \\
\hline Picture vocabulary & $\begin{array}{l}\text { Language vocabulary and } \\
\text { comprehension }\end{array}$ & \\
\hline
\end{tabular}

assessed with tasks of spatial memory, working memory, processing speed, executive function and attention.

QoL was assessed with the Functional Assessment of Cancer Therapy: Brain Cancer (FACT-Br, http://www.facit.org/ FACITOrg/Questionnaires). The FACT-Br includes sub-scores for physical wellbeing, social wellbeing, emotional wellbeing, and functional wellbeing (which make up the FACT-G general cancer sub-score), cognition (brain cancer sub-score), and FACT-Br total scores (32). FACT-G scores and sub-scores were compared to norms reported by Holzner et al. (32). Published norms are not available for the Brain Cancer sub-score.

\section{Imaging}

Patients underwent standard of care MR imaging of the brain on a 3T MR scanner (GE Healthcare, Waukesha, WI, USA) with an 8- or 32-channel head coil within 1 month of the cognitive and QoL assessment (usually on the same day). The MR imaging protocol included standard of care T2weighted Fluid Attenuated Inversion Recovery (FLAIR) and T1weighted pre- and post-gadolinium sequences. Each patient's previous pre-surgical and post-surgical MRIs were also included for evaluation. Hyperintensity volumes were measured on T2 FLAIR images (only 1 patient had residual T1-weighted contrast enhancement). The T2 FLAIR hyperintensity volumes were defined using semi-automated software (3D Slicer 4; http:// www.slicer.org) to include all the T2 FLAIR hyperintensity, relative to the surrounding normal tissue (Figure 1). Each volume was defined by a single investigator (TLL, AJ), with the guidance of a neuroradiologist (JEVM). The pre-surgical T2 FLAIR hyperintensity volume was used to determine the volume of tumor prior to the most recent surgery. The difference in volume between the pre- and post-surgical T2 FLAIR hyperintensity volumes was used to determine the Extent of Resection (EOR), and the change in T2 FLAIR hyperintensity volume from the post-surgical MRI to the time of cognitive and QoL assessment was used to measure tumor growth. Tumor location was categorized by hemisphere and primary lobe.

\section{Clinical and Demographic Variables}

Clinical and demographic data were collected by review of patients' medical records including age, education, time since diagnosis, previous treatment, tumor grade, KPS and use of anti-epileptic drugs (AEDs). Patients were classified according to the 2021 WHO integrated diagnoses of lower-grade glioma subgroups: astrocytoma, IDH mutated; astrocytoma, IDH wildtype; oligodendroglioma, IDH mutated and $1 \mathrm{p} 19 \mathrm{q}$ co-deleted; and NOS (molecular status unknown) (33). IDH mutation status and co-deletion of $1 \mathrm{p} 19 \mathrm{q}$ were determined by immunohistochemistry and FISH, respectively (34).

\section{Statistical Analysis}

The relationships between MR imaging characteristics, cognitive function and QoL scores were determined with ANOVAs and Spearman's rank correlations using JMP Pro 14 SAS software (http://www.jmp.com). Univariate analyses were performed for all variables. MR imaging characteristics included T2 FLAIR hyperintensity volume prior to surgery, T2 FLAIR hyperintensity volume at time of cognitive testing, EOR, T2 FLAIR hyperintensity growth, and tumor location (hemisphere and lobe). Clinical factors included age, WHO 2021 diagnosis, use of AEDs, prior chemotherapy, and prior combined radiation and chemotherapy. To correct for the 14 univariate analyses completed for all cognitive and QoL outcomes, we used the Benjamin-Hochberg False Discovery Rate $(F D R=0.2)$ threshold correction (35). Multivariate linear regression analyses were used to examine interactive effects of T2 FLAIR hyperintensity volume and clinical factors on cognition and QoL. These were exploratory analyses, given the small sample size, and no corrections were made for multiple comparisons.

\section{RESULTS}

\section{Patient Demographics and Clinical Factors}

Thirty patients with histologically confirmed LrGG were enrolled. Complete cognitive function data were collected for 27 patients and complete QoL data were collected for 28, due to equipment errors (the missing cognitive and QoL data were from different patients). Eighteen patients were diagnosed as WHO grade 2, and 12 patients were diagnosed as WHO grade 3. Twelve were diagnosed as astrocytoma, $I D H$ mutated; 14 as oligodendroglioma, $I D H$ mutated and $1 \mathrm{p} 19 \mathrm{q}$ codeleted; and four as NOS (owing to unknown IDH or $1 \mathrm{p} 19 \mathrm{q}$ status). Median age at enrollment was 45 years old (range 26-66) and median time from diagnosis was 72 months (range 20-204). Twenty-two patients had 1 previous surgery, 3 patients had 2 previous surgeries, 3 patients had 3 previous surgeries, and 2 patients had only previous biopsies (due to tumor location). Seventeen patients had received combined radiation and chemotherapy, 7 chemotherapy alone, and 6 neither. Twenty-one patients were on AEDs at the time of testing. No patients reported seizures in the last 6 months. Additional clinical characteristics and demographics are reported in Table 2. 


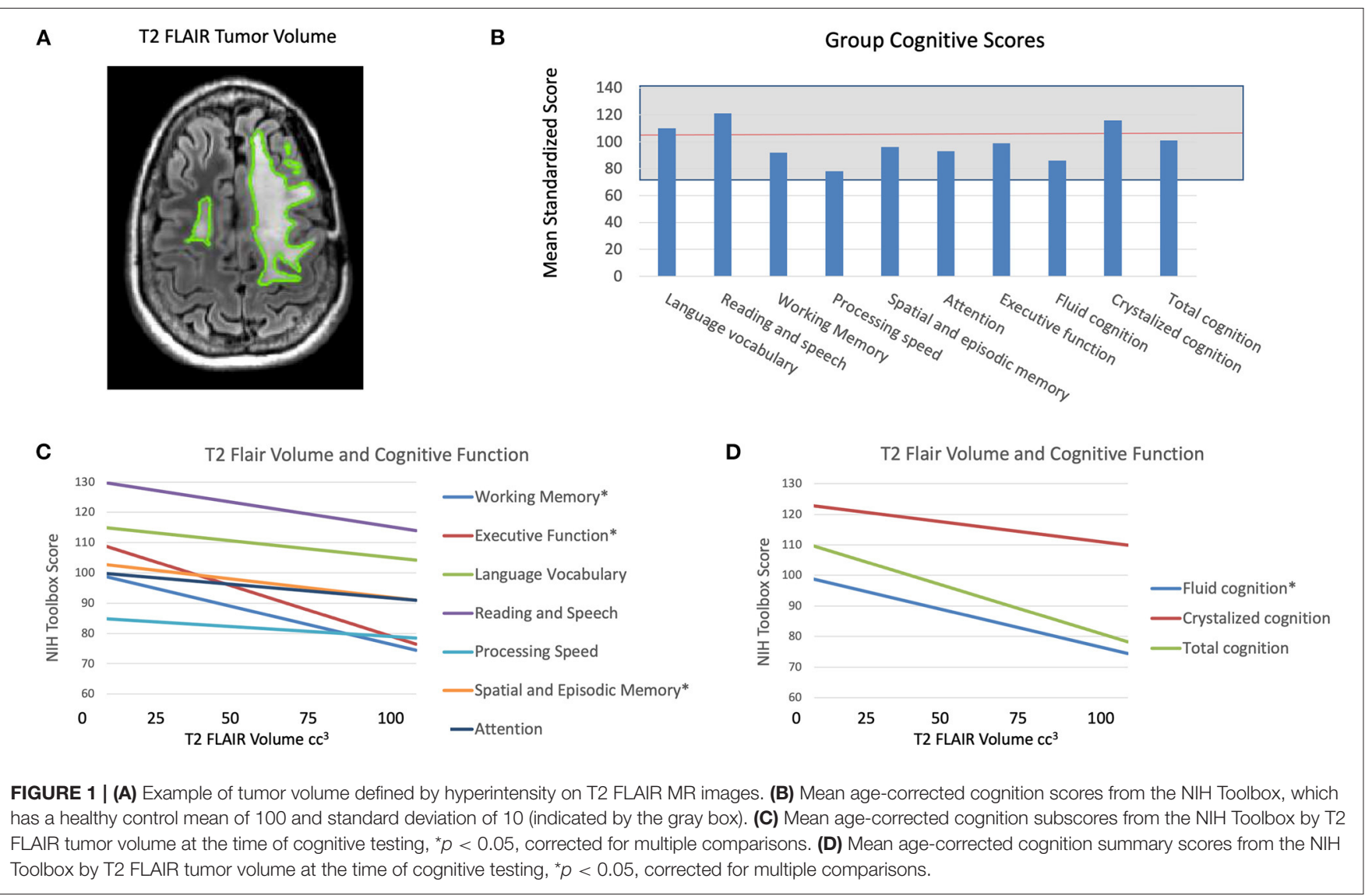

\section{Imaging Characteristics}

At the time of testing, T2 FLAIR hyperintensity volume mean (range) was $26.7 \mathrm{cc}^{3}$ (0.1-96). T2 FLAIR hyperintensity presurgery volume mean was $41.9 \mathrm{cc}^{3}(1-147.9)$, volumetric EOR mean was $74 \%(0-100 \%$, where $0=$ biopsy), and mean $\mathrm{T} 2$ FLAIR hyperintensity volume growth from the post-surgical MRI to the time of the cognitive and QoL assessment was $15.1 \mathrm{cc}^{3}$ (-35.7-68.7). Two patients had bilateral tumors, 17 patients had left hemisphere tumors, and 11 patients had right hemisphere tumors. Twenty were frontal, 7 were parietal, 2 were temporal, and 1 was occipital (Table 3 ).

Previous combined radiation and chemotherapy was significantly associated with larger T2 FLAIR hyperintensity volume at time of testing $(\mathrm{F}=9.7, p=0.004)$, and T2 FLAIR hyperintensity volume growth $(\mathrm{F}=8.9, p=0.006)$. Imaging variables were not significantly associated with any other demographic or clinical variables.

\section{Cognitive Functioning}

For the patients as a group, Processing speed was $\geq-1$ SD below average (all NIH Toolbox standardized age-corrected scores have a mean of 100 and standard deviation of 15), with mean standard score $=78$ (range $40-111$ ). Sixteen patients $(53 \%)$ scored $\geq 1$ SD below average on Processing speed. No other measures were $\geq$ $-1 \mathrm{SD}$ below average for the group as a whole. The group Fluid cognition mean standard score was 86 (range 58-113) and the group Crystallized cognition mean standard score was 116 (range 75-141). Thirteen patients (43\%) scored $\geq 1$ SD below average on Fluid cognition. Twenty patients (67\%) scored $\geq 1$ SD below average on at least one test (Table 4, Supplementary Table 1, Figure 1).

\section{Larger T2 FLAIR Hyperintensity Volume Is Associated With Lower Cognitive Functioning}

Larger T2 FLAIR hyperintensity volume at time of testing was significantly negatively associated with age-corrected performance on several tests, including: (1) List Sorting Working Memory Test (working memory) ( $\mathrm{F}=9.87, p=0.004)$; (2) Dimensional Change Card Sort Test (attention and executive functions) ( $\mathrm{F}=4.67, p=0.05)$; (3) Fluid cognition sub-scores $(\mathrm{F}=8.6, p=0.007)$; and (4) Total cognition score $(\mathrm{F}=6.4, p$ $=0.019)$ (Figures 1B,C). There were no significant associations between cognitive function and most recent pre-surgical T2 FLAIR hyperintensity volumes or EOR. Larger T2 FLAIR hyperintensity growth was significantly associated with lower age-corrected performance on the List Sorting Working Memory Test $(\mathrm{F}=5.4, p=0.029)$ and Fluid cognition sub-scores $(\mathrm{F}=4.8$, $p=0.038)$. There were no significant associations between tumor location (hemisphere or lobe) and cognitive function. When controlling for treatment history, there remain significant effects of T2 FLAIR hyperintensity volume on the List Sorting Working 
TABLE 2 | Patient demographics and clinical factors.

\begin{tabular}{|c|c|}
\hline Median age, years (range) & $45(26-66)$ \\
\hline Median education, years (range) & $16(8-20)$ \\
\hline \multicolumn{2}{|l|}{ Gender, $n(\%)$} \\
\hline Male & $11(37)$ \\
\hline Female & $19(63)$ \\
\hline Median time since diagnosis, months (range) & $72(20-204)$ \\
\hline Median time since last surgery, months (range) & $43(8-139)$ \\
\hline \multicolumn{2}{|l|}{$\mathrm{KPS}, n(\%)$} \\
\hline 100 & $4(13)$ \\
\hline 90 & $15(50)$ \\
\hline 80 & $10(33)$ \\
\hline 70 & $1(3)$ \\
\hline \multicolumn{2}{|l|}{ Prior Treatment, $n(\%)$} \\
\hline Surgery only & $6(20)$ \\
\hline Surgery and chemotherapy & $7(23)$ \\
\hline Surgery, chemotherapy and radiation therapy & $17(57)$ \\
\hline $\begin{array}{l}\text { Median time since last chemotherapy, months } \\
\text { (range) }\end{array}$ & $30(6-98)$ \\
\hline $\begin{array}{l}\text { Median time since radiation therapy, months } \\
\text { (range) }\end{array}$ & $36(6-139)$ \\
\hline \multicolumn{2}{|l|}{ Anti-epileptic medication at testing, $n(\%)$} \\
\hline Yes & $21(70)$ \\
\hline No & $9(30)$ \\
\hline \multicolumn{2}{|l|}{ WHO 2021 diagnosis, $n$ (\%) } \\
\hline $\begin{array}{l}\text { Oligodendroglioma, IDH mutant, 1p19q } \\
\text { co-deleted }\end{array}$ & $14(47)$ \\
\hline Grade 2 & 10 \\
\hline Grade 3 & 4 \\
\hline Astrocytoma, IDH mutant & $12(40)$ \\
\hline Grade 2 & 7 \\
\hline Grade 3 & 5 \\
\hline NOS & $4(13)$ \\
\hline Grade 2 & 1 \\
\hline Grade 3 & 3 \\
\hline
\end{tabular}

Memory Test (working memory) ( $\mathrm{F}=8.95, p=0.007$ ), Fluid cognition sub-scores $(\mathrm{F}=6.19, p=0.02)$, and Total cognition score $(\mathrm{F}=5.85, p=0.025)$. There was significant interactive effect between treatment history and T2 FLAIR hyperintensity volume on the List Sorting Working Memory Test ( $\mathrm{F}=5.63, p$ $=0.011$ ). The association of larger T2 FLAIR volume in patients with worse working memory was in fact strongest in patients with no previous chemotherapy or radiation therapy. There were no other significant statistical interactions between the effects of imaging variables and clinical variables on cognitive function.

\section{Increasing Age and Molecular Subgroup Associated With Lower Cognitive Functioning}

Increasing age at testing significantly correlated with lower age-corrected performance on several tests, including: (1) Oral Reading Recognition Test (reading and speech) $(\mathrm{F}=10.7, p$ $=0.003$ ); (2) List Sorting Working Memory Test (working
TABLE 3 | Imaging characteristics.

\begin{tabular}{ll}
\hline Imaging measures & \\
T2 FLAIR hyperintensity volume pre-surgery & \\
(mean, range) & $41.9 \mathrm{cc}^{3}(1-147.9)$ \\
Extent of resection (mean, range) & \\
T2 FLAIR hyperintensity volume at testing & $74 \%(0-100 \%)$ \\
(mean, range) & $26.7 \mathrm{cc}^{3}(0.1-96)$ \\
T2 FLAIR hyperintensity volume growth (mean, & \\
range) & $15.1 \mathrm{cc}^{3}(-35.7-68.7)$ \\
Tumor location & \\
\multicolumn{1}{c}{ Bilateral $(n=2)$} & \\
Left hemisphere $(n=17)$ & \\
& Frontal $n=2$ \\
Right hemisphere $(n=11)$ & Frontal $n=10$ \\
& Parietal $n=5$ \\
& Temporal $n=2$ \\
& Frontal $n=8$ \\
& Parietal $n=2$ \\
& Occipital $n=1$
\end{tabular}

memory) $(\mathrm{F}=8.0, p=0.009)$; (3) Dimensional Change Card Sort Test (attention and executive functions) $(\mathrm{F}=8.19, p=0.009) ;(4)$ Fluid cognition sub-score ( $\mathrm{F}=4.5, p=0.045)$; (5) Crystallized cognition sub-score $(\mathrm{F}=6.3, p=0.02)$; and (6) Total cognition score $(\mathrm{F}=7.22, p=0.013)$. Older patients performed lower than younger patients in all these domains, even after accounting for normal age-related declines in performance (Figure 2).

Patients with astrocytoma, IDH mutated LrGGs had significantly worse age-corrected performance than patients with oligodendrogliomas, IDH mutated 1p19q co-deleted LrGGs on: (1) Oral Reading Recognition Test (reading and speech) $(\mathrm{F}=$ 5.02, $p=0.036)$; (2) Fluid cognition scores $(\mathrm{F}=5.44, p=0.03)$; 3) Crystallized cognition scores $(\mathrm{F}=7.67, p=0.012)$, and (4) Total cognition scores $(\mathrm{F}=9.63, p=0.006)$ (Figure 2$)$. There were no significant statistical interactions between the effects of WHO 2021 diagnosis and grade on cognitive scores. There were no significant relationships between cognitive performance, time from diagnosis, grade, AED use, or previous treatment history.

\section{Previous Treatment Associated With Lower QoL Scores}

Eleven patients (37\%) scored worse than 1 SD below normal on the FACT-G total. Twenty-eight patients (93\%) scored lower than 1 SD below normal on at least one subscale (all but 2 patients), including 25 patients on the functional wellbeing subscale (Table 4, Supplementary Table 2). There were no significant associations between T2 FLAIR hyperintensity volumes at testing, pre-surgical T2 FLAIR hyperintensity volumes, EOR, T2 FLAIR hyperintensity growth or lobe, and FACT-Br scores. There were no significant statistical interactions between the effects of imaging variables and clinical variables on FACT-Br scores.

Previous treatment with chemotherapy was associated with lower self-reported cognitive function scores $(\mathrm{F}=6.5, p=0.017)$ and lower FACT-Br total scores $(\mathrm{F}=4.3, p=0.049)$ (Figure 2). Previous treatment with combined radiation and chemotherapy was associated with lower self-reported cognition scores $(\mathrm{F}=$ $6.1, p=0.02)$ and social wellbeing scores $(\mathrm{F}=5.5, p=0.027)$. There were no significant relationships between QoL scores and 
TABLE 4 | Cognitive and quality of life scores.

\begin{tabular}{|c|c|c|c|c|c|}
\hline Cognitive domain and QoL scores & $\begin{array}{c}\text { Mean score } \\
\text { (range) }\end{array}$ & $\begin{array}{c}\text { T2 FLAIR volume } \\
\text { at testing }\end{array}$ & $\begin{array}{l}\text { Pre-surgical T2 } \\
\text { FLAIR volume }\end{array}$ & $\begin{array}{l}\text { Extent of } \\
\text { resection }\end{array}$ & $\begin{array}{c}\text { T2 FLAIR volume } \\
\text { growth }\end{array}$ \\
\hline Processing speed & $78(40-111)$ & $\begin{array}{c}F=3.5 \\
p=0.073\end{array}$ & $\begin{array}{c}F=0.005 \\
p=0.99\end{array}$ & $\begin{array}{l}F=2.9 \\
p=0.1\end{array}$ & $\begin{array}{l}\mathrm{F}=2.01 \\
p=0.16\end{array}$ \\
\hline Attention & $93(55-135)$ & $\begin{array}{c}F=3.7 \\
p=0.066\end{array}$ & $\begin{array}{l}F=0.1 \\
p=0.76\end{array}$ & $\begin{array}{l}F=0.1 \\
p=0.75\end{array}$ & $\begin{array}{c}F=3.4 \\
p=0.078\end{array}$ \\
\hline Working memory & $92(-114)$ & $\begin{array}{l}F=9.87 \\
p=0.004\end{array}$ & $\begin{array}{l}F=2.8 \\
p=0.11\end{array}$ & $\begin{array}{l}F=1.7 \\
p=0.2\end{array}$ & $\begin{array}{c}F=5.4 \\
p=0.029\end{array}$ \\
\hline Spatial and episodic memory & $96(72-146)$ & $\begin{array}{l}F=3.26 \\
p=0.084\end{array}$ & $\begin{array}{l}\mathrm{F}=1.27 \\
p=0.27\end{array}$ & $\begin{array}{l}F=2.7 \\
p=0.11\end{array}$ & $\begin{array}{l}\mathrm{F}=0.77 \\
p=0.39\end{array}$ \\
\hline Executive function & $99(57-134)$ & $\begin{array}{l}F=4.18 \\
p=0.05\end{array}$ & $\begin{array}{l}\mathrm{F}=0.22 \\
p=0.65\end{array}$ & $\begin{array}{c}F=0.06 \\
p=0.8\end{array}$ & $\begin{array}{l}F=2.8 \\
p=0.11\end{array}$ \\
\hline Language vocabulary & $110(74-140)$ & $\begin{array}{c}F=1.3 \\
p=0.256\end{array}$ & $\begin{array}{l}F=1.5 \\
p=0.23\end{array}$ & $\begin{array}{l}F=0.9 \\
p=0.35\end{array}$ & $\begin{array}{l}F=0.16 \\
p=0.69\end{array}$ \\
\hline Reading and speech & $121(76-150)$ & $\begin{array}{c}F=5.14 \\
p=0.033\end{array}$ & $\begin{array}{l}\mathrm{F}=1.7 \\
p=0.2\end{array}$ & $\begin{array}{l}F=3.5 \\
p=0.07\end{array}$ & $\begin{array}{l}F=1.6 \\
p=0.22\end{array}$ \\
\hline Fluid cognition & $86(58-113)$ & $\begin{array}{c}F=8.6 \\
p=0.007\end{array}$ & $\begin{array}{l}F=0.22 \\
p=0.64\end{array}$ & $\begin{array}{l}F=1.2 \\
p=0.28\end{array}$ & $\begin{array}{c}F=4.8 \\
p=0.038\end{array}$ \\
\hline Crystallized cognition & $116(75-141)$ & $\begin{array}{l}\mathrm{F}=2.7 \\
p=0.11\end{array}$ & $\begin{array}{l}F=1.46 \\
p=0.24\end{array}$ & $\begin{array}{l}F=1.8 \\
p=0.19\end{array}$ & $\begin{array}{l}\mathrm{F}=0.55 \\
p=0.47\end{array}$ \\
\hline Total cognition & $101(67-126)$ & $\begin{array}{c}F=6.4 \\
p=0.019\end{array}$ & $\begin{array}{l}\mathrm{F}=0.88 \\
p=0.36\end{array}$ & $\begin{array}{l}F=1.9 \\
p=0.18\end{array}$ & $\begin{array}{l}F=2.4 \\
p=0.13\end{array}$ \\
\hline $\begin{array}{l}\text { FACT functional } \\
\text { wellbeing }\end{array}$ & $13(7-23)$ & $\begin{array}{l}F=0.26 \\
p=0.62\end{array}$ & $\begin{array}{l}F=0.31 \\
p=0.58\end{array}$ & $\begin{array}{l}F=0.16 \\
p=0.69\end{array}$ & $\begin{array}{l}F=0.18 \\
p=0.68\end{array}$ \\
\hline $\begin{array}{l}\text { FACT physical } \\
\text { wellbeing }\end{array}$ & $23(14-28)$ & $\begin{array}{c}F=0.06 \\
p=0.8\end{array}$ & $\begin{array}{l}F=0.5 \\
p=0.49\end{array}$ & $\begin{array}{l}F=0.01 \\
p=0.98\end{array}$ & $\begin{array}{l}\mathrm{F}=0.27 \\
p=0.61\end{array}$ \\
\hline $\begin{array}{l}\text { FACT emotional } \\
\text { wellbeing }\end{array}$ & $18(7-25)$ & $\begin{array}{l}F=0.7 \\
p=0.41\end{array}$ & $\begin{array}{l}F=0.04 \\
p=0.84\end{array}$ & $\begin{array}{l}F=0.01 \\
p=0.99\end{array}$ & $\begin{array}{l}\mathrm{F}=0.72 \\
p=0.40\end{array}$ \\
\hline $\begin{array}{l}\text { FACT social } \\
\text { wellbeing }\end{array}$ & $20(2-28)$ & $\begin{array}{l}F=0.05 \\
p=0.82\end{array}$ & $\begin{array}{l}F=0.8 \\
p=0.38\end{array}$ & $\begin{array}{l}F=0.04 \\
p=0.84\end{array}$ & $\begin{array}{l}F=0.24 \\
p=0.63\end{array}$ \\
\hline FACT brain cancer (cognition) & $63(43-77)$ & $\begin{array}{l}F=1.23 \\
p=0.28\end{array}$ & $\begin{array}{l}F=0.52 \\
p=0.48\end{array}$ & $\begin{array}{l}F=0.35 \\
p=0.56\end{array}$ & $\begin{array}{l}F=3.3 \\
p=0.08\end{array}$ \\
\hline $\begin{array}{l}\text { Fact G } \\
\text { (general) }\end{array}$ & $74(57-91)$ & $\begin{array}{l}F=0.04 \\
p=0.85\end{array}$ & $\begin{array}{l}F=0.17 \\
p=0.68\end{array}$ & $\begin{array}{c}F=0.06 \\
p=0.8\end{array}$ & $\begin{array}{l}\mathrm{F}=0.13 \\
p=0.72\end{array}$ \\
\hline Fact Br total & $137(100-165)$ & $\begin{array}{l}F=0.26 \\
p=0.62\end{array}$ & $\begin{array}{l}F=0.39 \\
p=0.54\end{array}$ & $\begin{array}{l}F=0.04 \\
p=0.85\end{array}$ & $\begin{array}{l}F=0.59 \\
p=0.45\end{array}$ \\
\hline
\end{tabular}

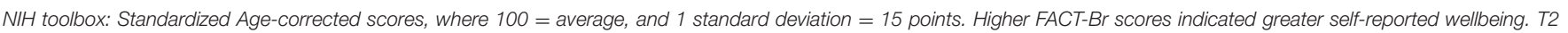
FLAIR Volume Growth = the change in T2 FLAIR hyperintensity volume from the post-surgical MRI to the time of cognitive and QoL assessment.

age, grade, AED use, time since diagnosis, WHO 2021 subgroup diagnosis. There were no significant correlations between FACT$\mathrm{Br}$ scores and NIH Toolbox Cognitive scores. This includes a lack of relationship between the FACT-BrC (self-reported cognition) and the objective NIH Toolbox cognitive measures, and between those measures and the FACT-BR emotional wellbeing scores (which includes questions about mood) and FACT-BR physical wellbeing scores (which includes questions about fatigue). However, there were significant correlations between between self-reported cognition (FACT-BrC) and physical wellbeing (Spearmans' rho $=0.64, p=0.003$ ) and between FACT-BrC and FACT-G scores (Spearmans' rho $=0.68, p=0.001$ ).

\section{DISCUSSION}

In this study, we evaluated objective cognitive test performance and QoL in 30 clinically stable LrGG patients who reported subjective cognitive concerns. The aim of this research was to identify imaging factors in clinically stable LrGG patients associated with cognitive and QoL impairments. Given that most patients demonstrated significant impairment in at least one cognitive and/or one QoL domain at a median of 6 years after diagnosis, this study highlights the continued importance of work in this area.

We identified imaging factors associated with lower cognitive function and QoL that may be useful when monitoring patients with these concerns. Larger T2 FLAIR hyperintensity volume at testing was associated with lower cognitive functioning in the domains of working memory, executive functioning, and processing speed, while performance on these domains did not correlate with pre-surgical tumor volume, or EOR. These associations were not explained by clinical factors such as treatment history, grade or molecular subgroup. The association with working memory was in fact strongest in patients with no previous chemotherapy or radiation therapy, indicating that it 


\section{A Age and Cognitive Function}

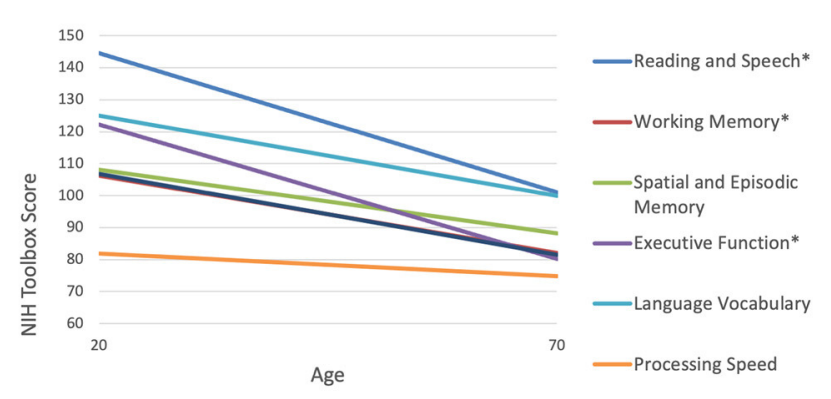

Age

C Treatment History and Quality of Life

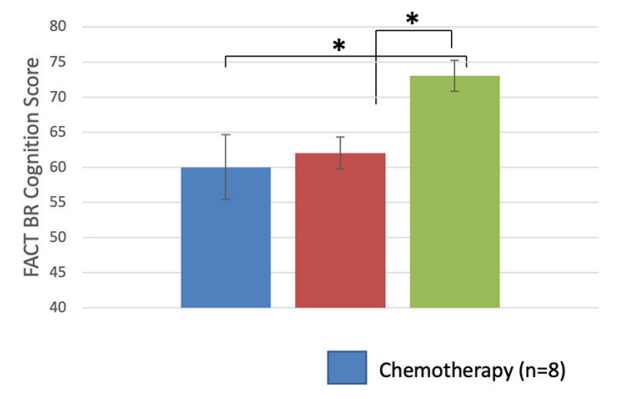

\section{B WHO 2016 Diagnosis and Cognitive Function}

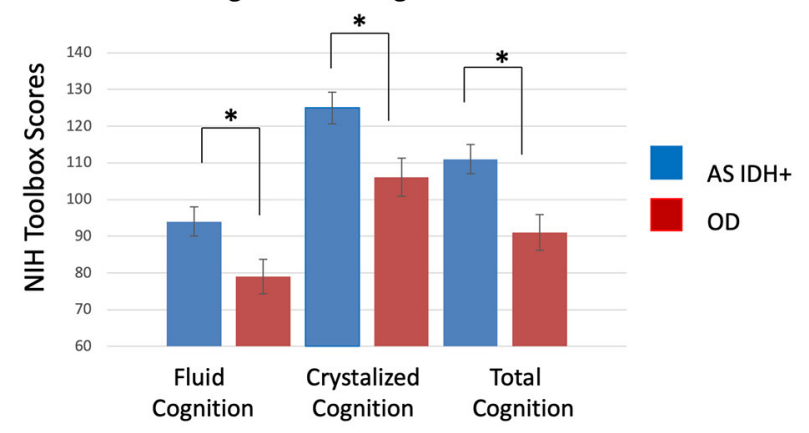

FIGURE 2 | (A) Mean age-corrected cognition subscores from the NIH Toolbox by patient age, * $p<0.05$, corrected for multiple comparisons. (B) Mean age-corrected cognition summary scores from the $\mathrm{NIH}$ Toolbox by WHO 2021 molecular diagnostic subgroup, ${ }^{*} p$ 0.05, corrected for multiple comparisons. (C) Mean FACT-BR cognition subscore, FACT-BR Total score by patient radiation and chemotherapy history, ${ }^{*} p<0.05$, corrected for multiple comparisons.

was not simply a by-product of the effects of radiation therapy, which is more likely to be used in patients with larger tumors. These significant associations are of particular interest as they are noted even on tests for which the group as a whole performed normally, indicating that individual differences in T2 FLAIR hyperintensity volumes vary with cognitive performance. Even in clinically stable patients, larger tumor volume may cause more disruption of functional networks, and alterations in structural and functional connectivity have been associated with cognitive function in glioma (36-41).

Older patients were more likely to have lower cognitive performance, even after accounting for normal age-related declines in performance, indicating that the cognitive impact of glioma increases with age. Patients with astrocytoma, IDH mutated LrGGs were more likely to have lower executive function scores and FACT-Br cognition scores than patients with oligodendroglioma, IDH mutated 1p19q-codeleted. Wefel et al. (42) found that IDH wild-type pre-surgical grade 3 and 4 glioma patients had lower cognitive scores than IDH mutated patients, and hypothesized this difference may be related to the more aggressive proliferation and dispersion characteristics of IDH wild-type tumors. Similar results have been reported by Derks et al. (43) and van Kessel et al. (25). Zhang et al. (44) incorporated $1 \mathrm{p} 19 \mathrm{q}$ and IDH mutation status to classify pre-surgical LrGG patients as astrocytoma, $I D H$ mutated or oligodendroglioma, IDH mutated 1p19qcodeleted and found, similar to the present study, that working memory scores were higher in the astrocytoma group than the oligodendroglioma group.

We also found that treatment with combined radiation and chemotherapy (compared to surgery only) was associated with worse self-reported QoL, including self-reported cognitive function. These results are consistent with reports that radiation therapy and chemotherapy can cause progressive cognitive decline $(23,45)$. Future studies with larger sample sizes will be necessary to determine the independent associations between cognitive function, molecular status and treatment history. However, even if the sample was large enough to include several potential covariates, it remains difficult to identify the separate contributions of these factors, because many treatment decisions are clinically driven by molecular status and grade.

It is also interesting to note which factors were not significantly associated with cognitive performance and QoL. Tumor laterality, for example, was not significantly associated with cognitive task performance, even for language tests. This suggests that these slow-growing gliomas impact broad functional networks, and that cognition may be preserved by compensatory re-organization during that period of slow growth. Although previous treatment with combined radiation and chemotherapy was associated with worse self-reported cognitive function, treatment was not associated with lower performance on cognitive tasks. Furthermore, there were no significant correlations between scores on cognitive tasks and self-reported QoL cognitive scores, indicating the complementary value 
of both types of assessment. The FACT-Br and other selfreport measures ask patients to limit their responses to their experience in a recent time window (e.g. last 7 days), which may not be reflective of their broader QoL experience. However, low associations between cognitive performance and selfperceived cognitive deficits have been reported across oncological populations, where self-perceived cognitive symptoms are more closely related to self-reported symptoms of physical and psychological distress, such as fatigue and depression (4649). Similarly, in our study, there were significant correlations between self-reported cognitive symptoms on the FACT-BrC and the physical wellbeing score and FACT-G score (summary of physical, emotional, social and functional wellbeing).

As mentioned above, sample size limits this study from examining the full effects of some factors, such as tumor location within hemisphere, and limits power to detect the main and interacting effects of the variables that were examined, such as WHO 2021 diagnosis, grade and treatment. Our patient sample is also biased both by the use of subjective cognitive concerns for study referral, and by the exclusion of patients with objective cognitive impairments so severe they cannot complete the assessments, and therefore our results may not reflect the associations between imaging factors, cognition and QoL in the total population of LrGG. The inclusion of cognition and QoL outcomes in future studies of clinically stable patients and in clinical trials of new therapeutic agents and treatment protocols will allow larger more representative patient groups, and longitudinal multifactorial analyses.

Given the emerging research for the use of cognitive rehabilitation for addressing cognitive and QoL concerns in LrGG (50-53), this study underscores the value of including cognitive and QoL assessments as part of LrGG care, as well as highlighting the groundwork needed to refine cognitive treatments. More specifically, while still in its infancy for use in LrGG, cognitive rehabilitation models may want to consider how clinical factors such as age, T2 FLAIR volumes, tumor molecular characteristics, and treatment effects may jointly influence treatment response.

In conclusion, it is important to appreciate that as LrGG becomes a disease with sustained periods of clinical stability, many of these patients are exhibiting significant impairments in cognitive function and QoL. We have found that larger current T2 FLAIR hyperintensity volume, older age, a diagnosis

\section{REFERENCES}

1. Chang SM, Cahill DP, Aldape KD, Mehta MP. Treatment of adult lower-grade glioma in the era of genomic medicine. Am Soc Clin Oncol Educ Book. (2016) 35:75-81. doi: 10.1200/EDBK_158869

2. Tucha O, Smely C, Preier M, Lange KW. Cognitive deficits before treatment among patients with brain tumors. Neurosurgery. (2000) 47:32433. doi: 10.1097/00006123-200008000-00011

3. Klein M, Heimans JJ, Aaronson NK, van der Ploeg HM, Grit J, Muller $M$, et al. Effect of radiotherapy and other treatment-related factors on mid-term to long-term cognitive sequelae in low-grade gliomas: a comparative study. Lancet. (2002) 360:1361-8. doi: 10.1016/S0140-6736(02) 11398-5 of astrocytoma, and adjuvant treatment are associated with lower cognitive functioning and QoL, and such clinical factors could help to identify LrGG patients in need of cognitive assessment and intervention.

\section{DATA AVAILABILITY STATEMENT}

The datasets presented in this article are not readily available because Data contain PHI. Requests to access the datasets should be directed to Tracy.Luks@ucsf.edu.

\section{ETHICS STATEMENT}

The studies involving human participants were reviewed and approved by University of California San Francisco IRB. The patients/participants provided their written informed consent to participate in this study.

\section{AUTHOR CONTRIBUTIONS}

TL, SC, KG, and JT designed the study. TL, JV-M, AJ, and JT carried out the data acquisition. TL analyzed the data. All authors participated in the interpretation of the results and the manuscript writing.

\section{FUNDING}

This research was funded by the American Cancer Society (to JT and TL); the LoGlio Collective (to all authors); the Sheri Sobrato Brisson Brain Cancer Fund (to CW-J, SC, and SH-J); Robert Wood Johnson Foundation [74259] (to SH-J); and National Institutes of Health [NINDS K08 110919-01] (to SH-J).

\section{SUPPLEMENTARY MATERIAL}

The Supplementary Material for this article can be found online at: https://www.frontiersin.org/articles/10.3389/fneur. 2021.769345/full\#supplementary-material

Supplemental Figure 1 | Age-corrected cognition subscores from the $\mathrm{NIH}$ Toolbox by patient age.

Supplementary Table 1 | Cognitive functioning.

Supplementary Table 2 | Quality of life. 
8. Noll KR, Bradshaw ME, Weinberg JS, Wefel JS. Relationships between neurocognitive functioning, mood, and quality of life in patients with temporal lobe glioma. Psychooncology. (2017) 26:617-24. doi: 10.1002/pon.4046

9. Cochereau J, Herbet G, Duffau H. Patients with incidental WHO grade II glioma frequently suffer from neuropsychological disturbances. Acta Neurochir. (2016) 158:305-12. doi: 10.1007/s00701-015-2674-3

10. Ek L, Kristoffersen Wiberg M, Vestberg S. Decline in executive functions and speed in suspected low-grade gliomas: A 3-year follow-up of a clinical cohort. Appl Neuropsychol Adult. (2018) 25:376-84. doi: 10.1080/23279095.2017.1316506

11. van Kessel E, Baumfalk AE, van Zandvoort MJE, Robe PA, Snijders TJ. Tumor-related neurocognitive dysfunction in patients with diffuse glioma: A systematic review of neurocognitive functioning prior to anti-tumor treatment. J Neurooncol. (2017) 134:9-18. doi: 10.1007/s11060-017-2503-Z

12. van Loon EMP, Heijenbrok-Kal MH, van Loon WS, van den Bent MJ, Vincent AJPE, de Koning I, et al. Assessment methods and prevalence of cognitive dysfunction in patients with low-grade glioma: a systematic review. J Rehabilit Med. (2015) 47:481-8. doi: 10.2340/16501977-1975

13. Boone M, Roussel M, Chauffert B, Le Gars D, Godefroy O. Prevalence and profile of cognitive impairment in adult glioma: a sensitivity analysis. $J$ Neurooncol. (2016) 129:123-30. doi: 10.1007/s11060-016-2152-7

14. Duffau H. Diffuse low-grade glioma, oncological outcome and quality of life: a surgical perspective. Curr Opin Oncol. (2018) 30:383-9. doi: 10.1097/CCO.0000000000000483

15. IJzerman-Korevaar M, Snijders TJ, de Graeff A, Teunissen SCCM, de Vos FYF. Prevalence of symptoms in glioma patients throughout the disease trajectory: a systematic review. J Neurooncol. (2018) 140:48596. doi: 10.1007/s11060-018-03015-9

16. Hoffermann M, Bruckmann L, Mahdy Ali K, Zaar K, Avian A, von Campe G. Pre- and postoperative neurocognitive deficits in brain tumor patients assessed by a computer based screening test. J Clin Neurosci. (2017) 36:316. doi: 10.1016/j.jocn.2016.10.030

17. Barzilai O, Ben Moshe S, Sitt R, Sela G, Shofty B, Ram Z. Improvement in cognitive function after surgery for low-grade glioma. J Neurosurg. (2019) 130:426-34. doi: 10.3171/2017.9.JNS17658

18. Rijnen SJM, Sitskoorn MM, Gehring K. Comment on: Effects of surgery on neurocognitive function in patients with glioma: a meta-analysis of immediate post-operative and long-term follow-up neurocognitive outcomes. J Neurooncol. (2019) 143:175-6. doi: 10.1007/s11060-019-03148-5

19. Reijneveld JC, Taphoorn MJB, Coens C, Bromberg JEC, Mason WP, HoangXuan K, et al. Health-related quality of life in patients with high-risk low-grade glioma (EORTC 22033-26033): A randomised, open-label, phase 3 intergroup study. Lancet Oncol. (2016) 17:1533-42. doi: 10.1016/S1470-2045(16)30305-9

20. Pulsifer MB, Duncanson H, Grieco J, Evans C, Tseretopoulos ID, MacDonald $\mathrm{S}$, et al. Cognitive and adaptive outcomes after proton radiation for pediatric patients with brain tumors. Int J Radiat Oncol Biol Phys. (2018) 102:3918. doi: 10.1016/j.ijrobp.2018.05.069

21. Dirven L, Reijneveld JC, Taphoorn MJB, Coens C, El-Badawy SA, Tzuk-Shina $\mathrm{T}$, et al. Impact of radiation target volume on health-related quality of life in patients with low-grade glioma in the 2-year period post treatment: a secondary analysis of the EORTC 22033-26033. Int J Radiat Oncol Biol Phys. (2019) 104:90-100. doi: 10.1016/j.ijrobp.2019.01.003

22. Cayuela N, Jaramillo-Jiménez E, Càmara E, Majós C, Vidal N, Lucas A, et al. Cognitive and brain structural changes in long-term oligodendroglial tumor survivors. Neuro Oncol. (2019) 21:1470-9. doi: 10.1093/neuonc/noz130

23. Makale MT, McDonald CR, Hattangadi-Gluth JA, Kesari S. Mechanisms of radiotherapy-associated cognitive disability in patients with brain tumours. Nat Rev Neurol. (2017) 13:52-64. doi: 10.1038/nrneurol.2016.185

24. Coomans MB, van der Linden SD, Gehring $\mathrm{K}$, Taphoorn MJB. Treatment of cognitive deficits in brain tumour patients: Current status and future directions. Curr Opin Oncol. (2019) 31:540-7. doi: 10.1097/CCO.0000000000000581

25. van Kessel E, Emons MAC, Wajer IH, van Baarsen KM, Broekman ML, Robe PA, et al. Tumor-related neurocognitive dysfunction in patients with diffuse glioma: A retrospective cohort study prior to antitumor treatment. Neuro-Oncol Pract. (2019) 6:463-72. doi: 10.1093/nop/npz008
26. Yoshida A, Motomura K, Natsume A, Chalise L, Iijima K, Hara D, et al. Preoperative predictive factors affecting return to work in patients with gliomas undergoing awake brain mapping. J Neurooncol. (2020) 146:195205. doi: 10.1007/s11060-019-03371-0

27. Rijnen SJM, Butterbrod E, Rutten G-JM, Sitskoorn MM, Gehring K. Presurgical identification of patients with glioblastoma at risk for cognitive impairment at 3-month follow-up. Neurosurgery. (2020) 87:1119-29. doi: 10.1093/neuros/nyaa190

28. Zelazo PD, Anderson JE, Richler J, Wallner-Allen K, Beaumont JL, Conway $\mathrm{KP}$, et al. NIH Toolbox Cognition Battery (CB): Validation of executive function measures in adults. J Int Neuropsychol Soc. (2014) 20:6209. doi: 10.1017/S1355617714000472

29. Lang S, Cadeaux M, Opoku-Darko M, Gaxiola-Valdez I, Partlo LA, Goodyear BG, et al. Assessment of cognitive, emotional, and motor domains in patients with diffuse gliomas using the national institutes of health toolbox battery. World Neurosurg. (2017) 99:448-56. doi: 10.1016/j.wneu.2016.12.061

30. Lang S, Gaxiola-Valdez I, Opoku-Darko M, Partlo LA, Goodyear BG, Kelly JJ, et al. Functional connectivity in frontoparietal network: indicator of preoperative cognitive function and cognitive outcome following surgery in patients with glioma. World Neurosurg. (2017) 105:91322e2. doi: 10.1016/j.wneu.2017.05.149

31. Heaton RK, Akshoomoff N, Tulsky D, Mungas D, Weintraub S, Dikmen $\mathrm{S}$, et al. Reliability and validity of composite scores from the NIH Toolbox Cognition Battery in adults. J Int Neuropsychol Soc. (2014) 20:58898. doi: 10.1017/S1355617714000241

32. Holzner B, Kemmler G, Cella D, De Paoli C, Meraner V, Kopp M, et al. Normative data for functional assessment of cancer therapy General scale and its use for the interpretation of quality of life scores in cancer survivors. Acta Oncol. (2004) 43:153-60. doi: 10.1080/02841860310023453

33. Louis DN, Perry A, Wesseling P, Brat DJ, Cree IA, Figarella-Branger D, et al. The 2021 WHO classification of tumors of the central nervous system: a summary. Neuro Oncol. (2021) 23:1231-51. doi: 10.1093/neuonc/noab106

34. Luks TL, McKnight TR, Jalbert LE, Williams A, Neill E, Lobo KA, et al. Relationship of in vivo MR parameters to histopathological and molecular characteristics of newly diagnosed, nonenhancing lower-grade gliomas. Transl Oncol. (2018) 11:941-9. doi: 10.1016/j.tranon.2018.05.005

35. McDonald J. Handbook of Biological Statistics (3rd ed.). (2015).

36. Jütten K, Mainz V, Gauggel S, Patel HJ, Binkofski F, Wiesmann M, et al. Diffusion tensor imaging reveals microstructural heterogeneity of normalappearing white matter and related cognitive dysfunction in glioma patients. Front Oncol. (2019) 9. doi: 10.3389/fonc.2019.00536

37. Jütten K, Mainz V, Delev D, Gauggel S, Binkofski F, Wiesmann M, et al. Asymmetric tumor-related alterations of network-specific intrinsic functional connectivity in glioma patients. Hum Brain Mapp. (2020) 41:454961. doi: 10.1002/hbm.25140

38. Liu Y, Hu G, Yu Y, Jiang Z, Yang K, Hu X, et al. Structural and functional reorganization within cognitive control network associated with protection of executive function in patients with unilateral frontal gliomas. Front Oncol. (2020) 10:794. doi: 10.3389/fonc.2020.00794

39. Lizarazu M, Gil-Robles S, Pomposo I, Nara S, Amoruso L, Quiñones I, et al. Spatiotemporal dynamics of postoperative functional plasticity in patients with brain tumors in language areas. Brain Lang. (2020) 202:104741. doi: 10.1016/j.bandl.2019.104741

40. Schouwenaars IT, de Dreu MJ, Rutten G-JM, Ramsey NF, Jansma JM. A functional MRI study of presurgical cognitive deficits in glioma patients. Neuro-Oncol Pract. (2021) 8:81-90. doi: 10.1093/nop/npaa059

41. Yuan B, Zhang N, Yan J, Cheng J, Lu J, Wu J. Resting-state functional connectivity predicts individual language impairment of patients with left hemispheric gliomas involving language network. NeuroImage Clin. (2019) 24:102023. doi: 10.1016/j.nicl.2019.102023

42. Wefel JS, Noll KR, Rao G, Cahill DP. Neurocognitive function varies by IDH1 genetic mutation status in patients with malignant glioma prior to surgical resection. Neuro Oncol. (2016) 18:1656-63. doi: 10.1093/neuonc/now165

43. Derks J, Kulik S, Wesseling P, Numan T, Hillebrand A, van Dellen E, et al. Understanding cognitive functioning in glioma patients: the relevance of IDH-mutation status and functional connectivity. Brain Behav. (2019) 9:e01204. doi: 10.1002/brb3.1204 
44. Zhang Z, Jin Z, Yang X, Zhang L, Zhang Y, Liu D, et al. Pre-operative neurocognitive function was more susceptible to decline in isocitrate dehydrogenase wild-type subgroups of lower-grade glioma patients. Front Neurol. (2020) 11:1667. doi: 10.3389/fneur.2020.591615

45. Greene-Schloesser D, Robbins ME. Radiation-induced cognitive impairment-from bench to bedside. Neuro Oncol. (2012) 14:iv3744. doi: 10.1093/neuonc/nos196

46. Bray VJ, Dhillon HM, Vardy JL. Systematic review of self-reported cognitive function in cancer patients following chemotherapy treatment. J Cancer Survivorship. (2018) 12:537-59. doi: 10.1007/s11764-0180692-x

47. Jung MS, Zhang M, Askren MK, Berman MG, Peltier S, Hayes DF, et al. Cognitive dysfunction and symptom burden in women treated for breast cancer: a prospective behavioral and fMRI analysis. Brain Imaging Behav. (2017) 11:86-97. doi: 10.1007/s11682-016-9507-8

48. Hutchinson AD, Hosking JR, Kichenadasse G, Mattiske JK, Wilson C. Objective and subjective cognitive impairment following chemotherapy for cancer: a systematic review. Cancer Treat Rev. (2012) 38:92634. doi: 10.1016/j.ctrv.2012.05.002

49. van Lonkhuizen PJC, Klaver KM, Wefel JS, Sitskoorn MM, Schagen SB, Gehring K. Interventions for cognitive problems in adults with brain cancer: a narrative review. Eur J Cancer Care. (2019) 28:e13088. doi: 10.1111/ecc. 13088

50. Weyer-Jamora C, Brie MS, Luks TL, Smith EM, Braunstein SE, VillanuevaMeyer JE, et al. Cognitive impact of lower grade gliomas and strategies for rehabilitation. Neuro-Oncol Pract. (2020) 8:117-28. doi: 10.1093/nop/ npaa072

51. Weyer-Jamora C, Brie MS, Luks TL, Smith EM, Hervey-Jumper SL, Taylor JW. Postacute cognitive rehabilitation for adult brain tumor patients. Neurosurgery. (2021) 89:945-53. doi: 10.1093/neuros/ nyaa552

52. Gehring K, Sitskoorn MM, Gundy CM, Sikkes SAM, Klein M, Postma TJ, et al. Cognitive rehabilitation in patients with gliomas: a randomized, controlled Trial. J Clin Oncol. (2009) 27:3712-22. doi: 10.1200/JCO.2008.20.5765

53. Locke DEC, Cerhan JH, Wu W, Malec JF, Clark MM, Rummans TA, et al. Cognitive rehabilitation and problem- solving to improve quality of life of patients with primary brain tumors: a pilot study. J Support Oncol. (2008) 6:383-91.

Conflict of Interest: The authors declare that the research was conducted in the absence of any commercial or financial relationships that could be construed as a potential conflict of interest.

Publisher's Note: All claims expressed in this article are solely those of the authors and do not necessarily represent those of their affiliated organizations, or those of the publisher, the editors and the reviewers. Any product that may be evaluated in this article, or claim that may be made by its manufacturer, is not guaranteed or endorsed by the publisher.

Copyright (C 2022 Luks, Villanueva-Meyer, Weyer-Jamora, Gehring, Jakary, HerveyJumper, Braunstein, Bracci, Brie, Smith, Chang and Taylor. This is an open-access article distributed under the terms of the Creative Commons Attribution License (CC $B Y)$. The use, distribution or reproduction in other forums is permitted, provided the original author(s) and the copyright owner(s) are credited and that the original publication in this journal is cited, in accordance with accepted academic practice. No use, distribution or reproduction is permitted which does not comply with these terms. 